Article

\title{
Outbreak of Porcine Reproductive and Respiratory Syndrome Virus 1 in Taiwan
}

\author{
Wei-Hao Lin $1,2,+\oplus$, Kraijak Kaewprom ${ }^{2,3,+}$, Sheng-Yuan Wang ${ }^{1}$, Chuen-Fu Lin ${ }^{4}$, \\ Cheng-Yao Yang ${ }^{5}$, Ming-Tang Chiou ${ }^{1,2, *}$ and Chao-Nan Lin ${ }^{1,2, *}$ \\ 1 Animal Disease Diagnostic Center, College of Veterinary Medicine, National Pingtung University of Science \\ and Technology, Pingtung 91201, Taiwan; j7967633@gmail.com (W.-H.L.); april89000@gmail.com (S.-Y.W.) \\ 2 Department of Veterinary Medicine, College of Veterinary Medicine, National Pingtung University of \\ Science and Technology, Pingtung 91201, Taiwan; addyvm.rmu@gmail.com \\ 3 Program of Veterinary Technology, Faculty of Agricultural Technology, Rajabhat Mahasarakham University, \\ Mahasarakham 44000, Thailand \\ 4 Department of Veterinary Medicine, College of Veterinary Medicine, National Chiayi University, Chiayi \\ 60054, Taiwan; linchuenfu@gmail.com \\ 5 Graduate Institute of Veterinary Pathobiology, College of Veterinary Medicine, National Chung Hsing \\ University, Taichung 40227, Taiwan; yangchengyao@nchu.edu.tw \\ * Correspondence: mtchiou@mail.npust.edu.tw (M.-T.C.); cnlin6@mail.npust.edu.tw (C.-N.L.); \\ Tel.: +886-8-7703202-5057 (M.-T.C.); +886-8-7703202-5047 (C.-N.L.) \\ + These authors contributed equally to this work.
}

Received: 4 February 2020; Accepted: 14 March 2020; Published: 16 March 2020

\begin{abstract}
Porcine reproductive and respiratory syndrome (PRRS) causes significant economic losses in the swine industry worldwide. The PRRS virus (PRRSV) can be divided into two species, PRRSV 1 (European) and PRRSV 2 (North American). In Taiwan, PRRSV 2 isolates are dominant and cause respiratory symptoms in nursing pigs. From October to November 2018, in a pig herd in central Taiwan, pregnant sows had abortions and stillbirths, and piglets suffered from respiratory disorders. Laboratory tests identified the presence of PRRSV 1 in serum from sows and suckling piglets in this scenario. The complete genome of the identified PRRSV 1 strain was genetically closely related to that of a European PRRSV vaccine strain (98.2\%). This local European isolate is designated as PRRSV/NPUST-2789-3W-2/TW/2018 (NPUST2789). This report is the first to indicate an outbreak in Taiwan of a PRRSV 1 strain that shares a common evolutionary ancestor with the European PRRSV vaccine strain.
\end{abstract}

Keywords: porcine reproductive and respiratory syndrome virus 1; European type; Taiwan

\section{Introduction}

Porcine reproductive and respiratory syndrome (PRRS) is one of the most economically devastating diseases facing the pork industry worldwide. The causative agent, the PRRS virus (PRRSV), was identified in the early 1990s [1,2]. A retrospective analysis revealed the presence of PRRSV antibodies in Canadian samples as early as 1979 [3]. PRRSV is an enveloped, positive-stranded RNA virus with a genome measuring approximately $15 \mathrm{~kb}$. Recently, PRRSV was reclassified to the genus Betaarterivirus and belongs to the family Arteriviridae within the order Nidovirales, which is divided into two species, Betaarterivirus suid 1 (PRRSV 1, European) and Betaarterivirus suid 2 (PRRSV 2, North American) [4]. There is approximately 60\% genomic sequence identity between the PRRSV 1 and PRRSV 2 strains [5-7].

During PRRSV infection, clinical disease is detectable in pigs of all of ages [8]. The clinical presentation of PRRSV can range from asymptomatic to devastating, with symptoms such as anorexia, high fever, hyperpnea, abortion, stillbirth or death in sows [8]. Suckling piglets are often infected by 
PRRSV through vertical or horizontal transmission. PRRSV infection in suckling piglets is characterized most consistently by listlessness, emaciation, splay leg posture, hyperpnea, dyspnea, and increased preweaning mortality [8]. Although more than 30 years have passed since PRRSV was first described, current management strategies mainly focus on preventing PRRSV infection using vaccination [9]. However, the currently available modified live vaccines (MLVs) are not sufficient to eliminate the virus [9]. The MLV vaccine can elicit not only humoral but also cell-medicated immune responses against PRRSV. In addition to efficacy, reverse virulence of MLV [10], recombination between wild-type PRRS and MLV [11-13] or recombination between different MLVs had been reported [14,15]. Therefore, the safety and cross-protection of MLV warrant further investigation.

In Asia, PRRSV 1 and PRRSV 2 have been reported to exist in several countries, including China, Japan, South Korea, Thailand and Vietnam [16-22]. In Taiwan, PRRSV 2 is dominant, while PRRSV 1 still exists but rarely causes significant economic losses [23]. In the present study, we investigated the previously unidentified PRRSV 1 outbreak in Taiwan. We reported a genetic and phylogenetic analysis of the PRRSV 1 isolate, designated PRRSV/NPUST-2789-3W-2/TW/2018 (NPUST2789), which is genetically closely related to that of a European PRRSV vaccine strain.

\section{Materials and Methods}

\subsection{Clinical Cases}

From October to November 2018, pregnant sows had abortions and stillbirths, and piglets suffered from respiratory disorders in a 550-sow farrow-to-finish pig farm located in central Taiwan. The farm has good external biosecurity and is free of porcine epidemic diarrheal virus. Neither sows nor piglets exhibited clinical signs of PRRS without PRRSV vaccination in this farm, which had been classified as positive stable and monitored by the Animal Disease Diagnostic Center (ADDC), National Pingtung University of Science of Technology (NPUST) [24].

\subsection{Sample Preparation and Quantification of PRRSV RNA}

Blood samples were collected from the affected animals and submitted to the ADDC, NPUST. Total nucleic acids were extracted from serum samples using a MagNA Pure LC total nucleic acid isolation kit (Roche Diagnostics GmbH, Mannheim, Germany) operated on a MagNA Pure 24 System (Roche Applied Science, Rotkreuz, Switzerland) according to the manufacturer's protocol. All samples were tested for PRRSV by a modified PRRSV real-time PCR assay [25]. The primers PRRSV-M177F (5'-CATTCTGGCCCCTGCCCA-3'), PRRSV M177R (5'-ACCACTCCYYGYTTDACAGCT- $\left.{ }^{\prime}\right)$, the PRRSV 1 zip nucleic acid (ZNA) probe (HEX-CGCTGTGAGAAAGCCCGG-ZNA4 BHQ1) and PRRSV 2 ZNA probe (FAM-CTCGTGTTGGGTGGCAGA-ZNA4 BHQ1), which targets the M gene, were used for quantitative real-time polymerase chain reaction to detect PRRSV in the clinical specimens.

\subsection{Serology}

All serum samples were tested for the presence of anti-PRRSV antibodies using a commercial ELISA kit (IDEXX PRRS X3 Ab Test; IDEXX Laboratories Inc, Westbrook, ME, USA) according to the manufacturer's instructions. Serum samples were considered positive for the presence of PRRSV antibody when the average sample-to-positive $(\mathrm{S} / \mathrm{P})$ ratio was $\geq 0.4$.

\subsection{Virus Isolation and Whole Genome Sequencing of PRRSV}

To harvest the highest viral load for whole genome sequencing of PRRSV, $0.5 \mathrm{~mL}$ of PRRSV-positive serum was added to Meat Animal Research Center-145 (MARC-145) cells and observed daily for cytopathic effects. Nucleic acids were extracted from the culture medium using a MagNA Pure 24 System (Roche Applied Science, Rotkreuz, Switzerland). The nucleic acids were end-repaired, A-tailed and adapter-ligated following the Illumina TruSeq DNA preparation protocol. After library 
construction, it was used for next-generation sequencing (NGS) by a NovaSeq 6000 System (Illumina, San Diego, CA, USA).

\subsection{Open Reading Frame 5 Amplification and Sequencing}

The open reading frame 5 (ORF5) genes were amplified and sequenced as described in a previous study [26]. The nucleotide sequences were determined from both orientations by an ABI 3730XL DNA analyzer (Applied Biosystems, Foster City, CA).

\subsection{Sequence and Phylogenetic Analysis}

The complete sequences of local PRRSV were then compared with other reference PRRSV strains, and the results are summarized in Table 1. Multiple alignments of nucleic acid sequences were performed by the Clustal W methods in Molecular Evolutionary Genetics Analysis X software (MEGA $X)$ [27]. Phylogenetic trees were constructed using the maximum likelihood method based on the Kimura 2-parameter model in MEGA X [27]. The sequence identity of nucleotides and amino acids was determined by MegAlign software (Lasergene, DNASTAR, Madison, WI, USA). The similarity versus position was plotted by SimPlot v3.5.1 software within a 200-bp window sliding along the genome alignment (20-bp step size) [28].

Table 1. Sequence identity of PRRSV/NPUST-2789-3W-2/TW/2018 and the reference strains.

\begin{tabular}{|c|c|c|c|c|c|c|}
\hline \multirow[b]{2}{*}{ Virus } & \multirow[b]{2}{*}{ Species } & \multirow[b]{2}{*}{ Year } & \multirow{2}{*}{$\begin{array}{l}\text { Country/ } \\
\text { Company }\end{array}$} & \multicolumn{2}{|c|}{ Sequence Identity (\%) } & \multirow{2}{*}{$\begin{array}{c}\text { GenBank } \\
\text { Accession } \\
\text { Number }\end{array}$} \\
\hline & & & & $\begin{array}{c}\text { Whole } \\
\text { Genome }\end{array}$ & ORF5 & \\
\hline Amervac PRRS ${ }^{1}$ & PRRSV 1 & $\mathrm{~N} / \mathrm{A}$ & HIPRA & 98.2 & 99.0 & GU067771 \\
\hline Porcilis PRRS & PRRSV 1 & N/A & MSD & 93.8 & 94.2 & KF991509 \\
\hline $\begin{array}{l}\text { Ingelvac } \\
\text { PRRSFLEX }\end{array}$ & PRRSV 1 & N/A & $\begin{array}{l}\text { Boehringer } \\
\text { Ingelheim }\end{array}$ & 87.4 & 92.6 & KT988004 \\
\hline Pyrsvac- $183^{1}$ & PRRSV 1 & N/A & SYVA & $\mathrm{N} / \mathrm{A}$ & 98.5 & DQ345726 \\
\hline Ingelvac PRRS 2 & PRRSV 2 & N/A & $\begin{array}{l}\text { Boehringer } \\
\text { Ingelheim }\end{array}$ & 57.8 & 59.7 & EF484033 \\
\hline Fostera PRRS ${ }^{2}$ & PRRSV 2 & N/A & Zoetis & 57.5 & 59.2 & AF494042 \\
\hline Lelystad virus & PRRSV 1 & 1991 & Netherlands & 94.1 & 94.5 & M96262 \\
\hline Lena & PRRSV 1 & 2007 & Belarus & 80.2 & 82.8 & JF802085 \\
\hline HUN60077/16 & PRRSV 1 & 2016 & Hungary & 91.7 & 98.2 & MK167464 \\
\hline NPUST-2789-3W-2 & PRRSV 1 & 2018 & Taiwan & 100 & 100 & MN242825 \\
\hline NPUST-2789-3W-5 & PRRSV 1 & 2018 & Taiwan & N/A & 99.8 & MN265857 \\
\hline NPUST-2860-S-6 & PRRSV 1 & 2018 & Taiwan & N/A & 99.8 & MN265858 \\
\hline VR2332 & PRRSV 2 & 1992 & USA & 57 & 59.9 & EF536003 \\
\hline MD001 & PRRSV 2 & 1991 & Taiwan & 56.1 & 58.4 & KP998431 \\
\hline MN184 & PRRSV 2 & 2001 & USA & 57.9 & 58.4 & EF484031 \\
\hline JXA1 & PRRSV 2 & 2006 & China & 57.3 & 60.7 & EF112445 \\
\hline $107-844$ & PRRSV 2 & 2018 & Taiwan & 57.8 & 56.8 & MK291410 \\
\hline
\end{tabular}

${ }^{1}$ Modified live attenuated PRRSV 1 vaccines were used in Taiwan. ${ }^{2}$ Modified live attenuated PRRSV 2 vaccines were used in Taiwan

\section{Results}

\subsection{Emergence of PRRSV 1}

A total of 70 sows were culled due to this outbreak, including 50 aborted sows, 5 sudden deaths of sows and 15 sows with severe anorexia. The suckling piglets were unthrifty and exhibited dyspnea and increased the preweaning mortality in this period. Forty percent of weaned pigs showed severe respiratory signs and 25\% mortality among the nursery piglets. In laboratory testing, 3 of 16 affected sows $\left(18.75 \%\right.$, viral load range from $1 \times 10^{1}$ to $1.3 \times 10^{5}$ genomes/ $\left.\mu \mathrm{L}\right)$ and 5 of 9 affected suckling piglets $\left(55.6 \%\right.$, viral load range from $3.6 \times 10^{4}$ to $1.1 \times 10^{6}$ genomes/ $\left.\mu \mathrm{L}\right)$ were positive for PRRSV 1 
(Figure 1). The average sample-to-positive (S/P) ratio of the PRRSV antibody of these sows was 2.54, ranging from 0.32 to 4.14 , and the positive rate was $87.5 \%$. Ten of 16 sows had $\mathrm{S} / \mathrm{P}$ ratios higher than 3.0, as determined using a commercial ELISA kit (Figure 1). Collectively, this farm suffered a PRRSV 1 outbreak, and this scenario is the first in Taiwan to be reported.

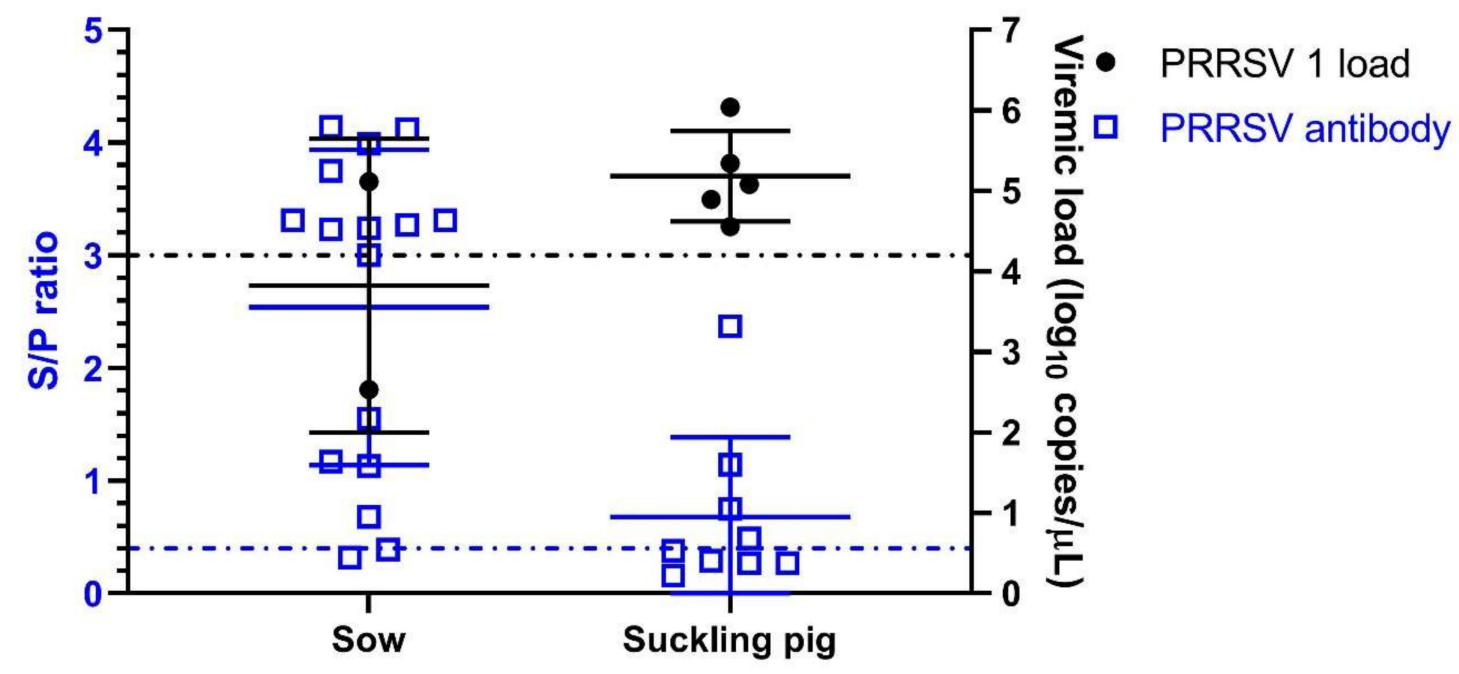

Figure 1. PRRSV 1 load and antibody level (sample-to-positive (S/P) ratio) of sows and suckling piglets suffered from porcine reproductive and respiratory syndrome (PRRS) in the present study.

\subsection{Phylogenetic Analysis of Whole Genome Sequences}

Viremic serum from a 3-week-old piglet (NPUST-2789-3W-2) was used for PRRSV isolation by Meat Animal Research Center-145 (MARC-145) cells (Figure 2). This isolate was denominated as PRRSV/NPUST-2789-3W-2/TW/2018 (NPUST2789). The full genomic RNA sequence of NPUST2789 (accession number: MN242825) comprises 15,111 nucleotides, excluding the 3' polyadenylated nucleotides. Similarity comparisons with SimPlot v3.5.1 revealed that the whole genome of NPUST2789 was most similar to that of the European PRRS vaccine Amervac PRRS (Figure 3). The entire genome of NPUST2789 was 98.2\% and 94.1\% similar to the European PRRS vaccine Amervac PRRS and prototype PRRSV 1 Lelystad virus, respectively (Table 1 and Figure S1). Phylogenetic trees based on the whole genomes and ORF 1a to ORF 7 nucleotide sequences showed the complete genome, and each ORF of NPUST2789 was most closely related to those of the known Amervac PRRS (Figure 4 and Figure S1).

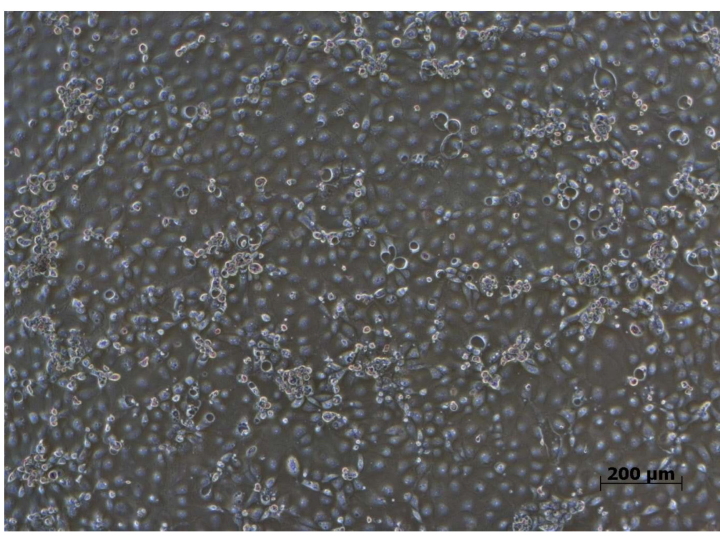

(a)

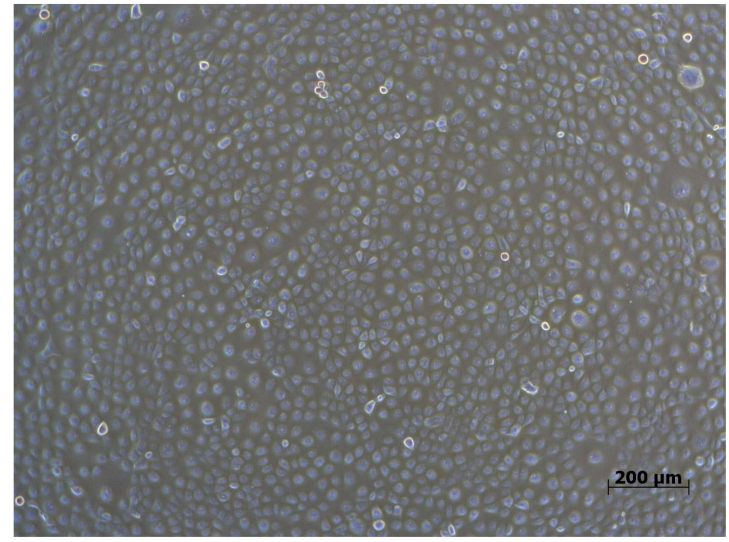

(b)

Figure 2. (a) Meat Animal Research Center-145 (MARC-145) cells cultured with serum from diseased suckling piglets. (b) Uninfected MARC-145 cells. 

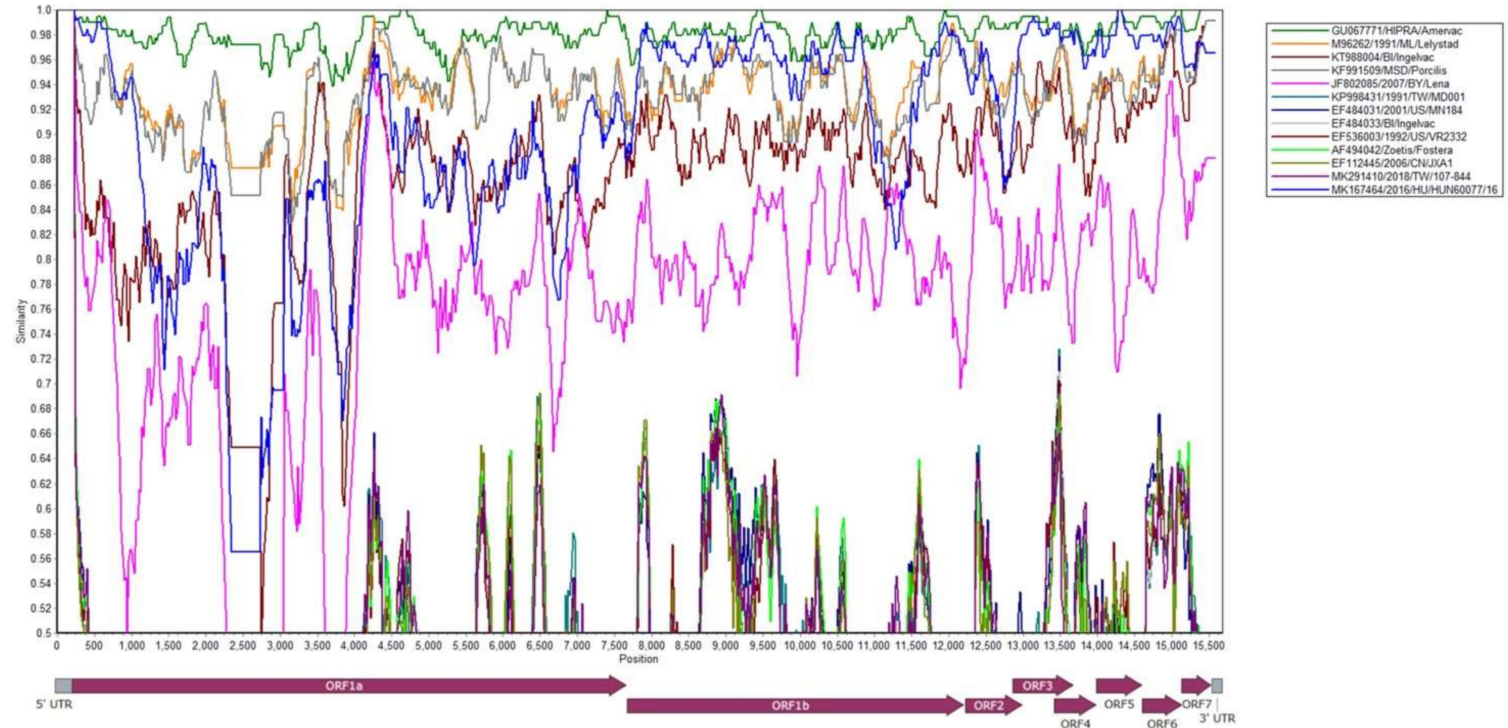

Figure 3. Nucleotide sequence similarity of the complete genome of PRRSV/NPUST-2789-3W-2/TW/2018 with that of other PRRSVs.
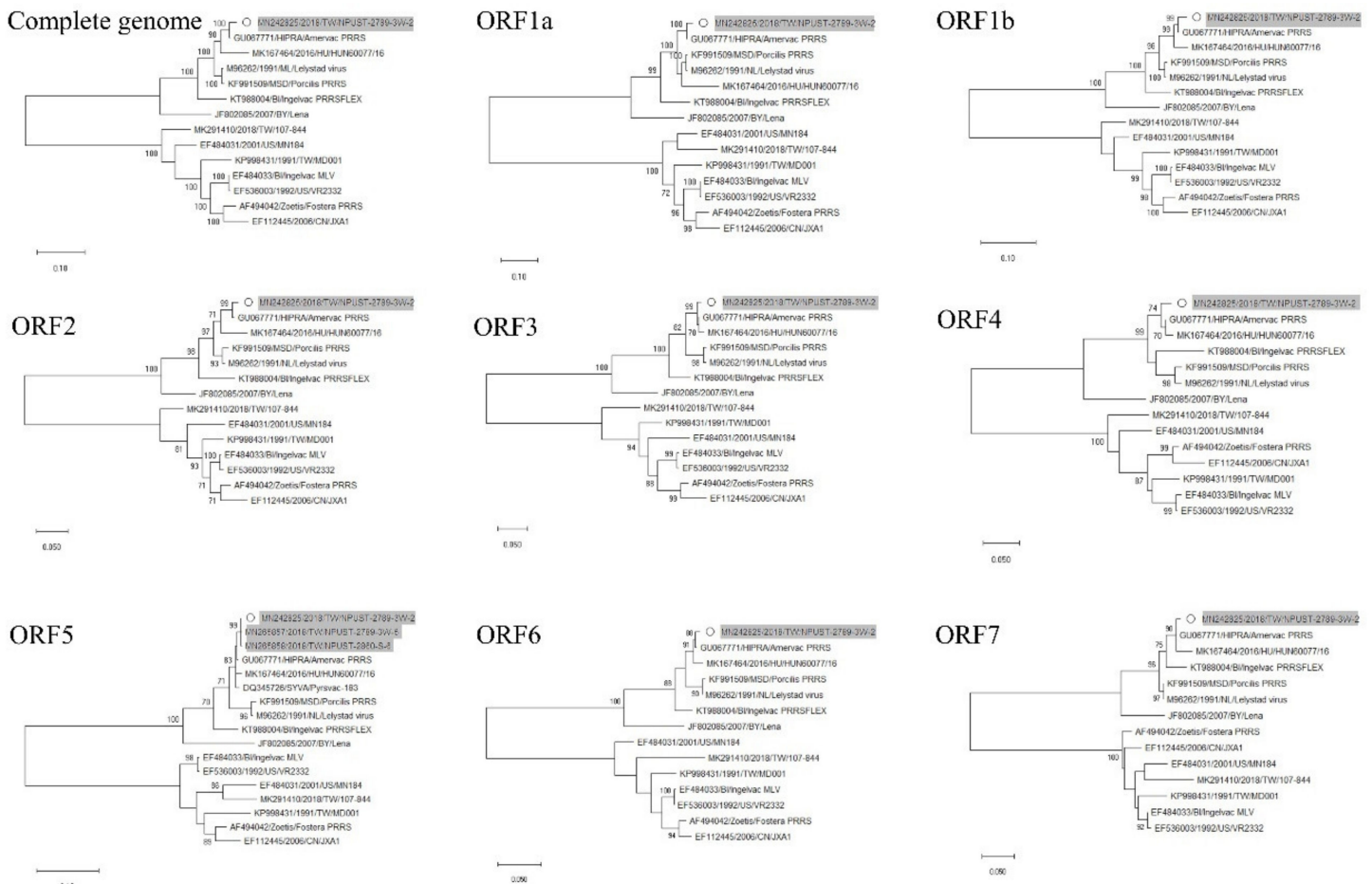

Figure 4. Phylogenetic relationships constructed using complete genomes, ORF1a, ORF1b, ORF2, ORF3, ORF4, ORF5, ORF6 and ORF7 gene sequences of PRRSV/NPUST-2789-3W-2/TW/2018 (NPUST2789) and other PRRSVs. The phylogenetic tree was constructed using the maximum likelihood method with bootstrap analysis $(n=1000)$ to evaluate clade confidence. Bootstrap support values greater than 70 are shown. The circle represents the PRRSV strain NPUST2789. The gray underlay represents local PRRSV 1 strains isolated in the present study.

\subsection{Phylogenetic, Amino Acid and Nucleotide Sequence Analysis of ORF5}

The ORF5 sequences from another 3-week-old piglet (NPUST-2789-3W-5, MN265856) and a viremic sow (NPUST-2860-S-6, MN265858) were almost identical to those of NPUST2789 (Figure 4, Table 1 and Figure S2). In contrast to the low nucleotide and amino acid sequence identities between other 
European PRRSV1 strains (Leylstad and HUN60077/16 strains) and Taiwanese PRRSV 1 NPUST2789, the sequence identity levels between this outbreak of PRRSV 1 isolates and Amervac PRRS appeared to be considerably higher (Table 2 and Figure S2). Taken together, these results confirmed that this outbreak of PRRSV 1 shared a common origin with Amervac PRRS.

Table 2. Sequence identities of nucleotide and amino acid sequences between PRRSV/NPUST2789-3W-2/TW/2018 and the reference strains.

\begin{tabular}{ccccccc}
\hline \multirow{2}{*}{ ORF } & \multicolumn{2}{c}{ Amervac PRRS } & \multicolumn{2}{c}{ Lelystad Virus } & \multicolumn{2}{c}{ HUN60077/16 } \\
\cline { 2 - 7 } & $\begin{array}{c}\text { Nucleotide } \\
\mathbf{( \% )}\end{array}$ & $\begin{array}{c}\text { Amino Acid } \\
\mathbf{( \% )}\end{array}$ & $\begin{array}{c}\text { Nucleotide } \\
\mathbf{( \% )}\end{array}$ & $\begin{array}{c}\text { Amino Acid } \\
\mathbf{( \% )}\end{array}$ & $\begin{array}{c}\text { Nucleotide } \\
\mathbf{( \% )}\end{array}$ & $\begin{array}{c}\text { Amino Acid } \\
\mathbf{( \% )}\end{array}$ \\
\hline ORF1a & 98.1 & 98.1 & 93.6 & 94.4 & 87.2 & 87.6 \\
ORF1b & 98.2 & 99.1 & 94.3 & 98.2 & 95.4 & 98.6 \\
ORF2 & 98.7 & 97.7 & 94.9 & 94.6 & 93.6 & 94.6 \\
ORF3 & 98.9 & 99.6 & 94.5 & 94.4 & 98 & 98.5 \\
ORF4 & 97.8 & 96.8 & 93.3 & 94.1 & 96.9 & 94.6 \\
ORF5 & 99.8 & 100 & 98.5 & 97.1 & 98.2 & 97.1 \\
ORF6 & 99.2 & 98.3 & 96.4 & 96.6 & 98.1 & 97.1 \\
ORF7 & 99.2 & 98.5 & 96.2 & 96.2 & 96.7 & 96.2 \\
\hline
\end{tabular}

\section{Discussion}

In the present study, we characterized a previously unidentified PRRSV 1 outbreak in Taiwan. A comparison of the data obtained was made with available sequences of entire PRRSV 1 . This comparison clearly indicated a close relationship between our PRRSV 1 and the vaccine virus Amervac PRRS and less close relationship to other PRRSV1 vaccines (Table 1). To date, there are four commercial PRRSV MLV vaccines available in Taiwan, including Ingelvac PRRS ${ }^{\circledR}$ MLV (PRRSV 2, Boehringer Ingelheim), Fostera ${ }^{\circledR}$ PRRS (PRRSV 2, Zoetis), Amervac PRRS (PRRSV 1, Hipra) and Pyrsvac-183 PRRS (PRRSV 1, Syva) (Table 1). Interestingly, for the last decade, this herd has not been immunized with any PRRSV 1 or PRRSV 2 vaccine, and the farmer has never introduced boars or gilts from domestic breeding herds. The Amervac PRRS MLV vaccine has been used in Taiwanese pig farms for almost ten years. Therefore, we speculated that PRRSV 1 transmits into pig farms by the adaptation of Amervac PRRS MLV. A similar finding had been observed in PRRSV 2 in 1998; PRRSV 2 in Danish pig farms was due to the spread of the vaccine virus [29]. Therefore, the prevalence of PRRSV 1 in Taiwan warrants further investigation.

PRRSV 1 vaccine-like cases have been reported recently in Hungary [11], Denmark [15] and China [30]. The genome of the Hungarian PRRSV 1 strain HUN60077/16, which has a mosaic structure of the genome, showed a notably close genetic relationship to the PRRSV 1 vaccine Unistrain, also known as Amervac PRRS in Taiwan or China. A similar report was also found in China; Amervac-like isolates carried out the genes from vaccine strains and circulating wild strains [30]. Certain issues need to be further investigated: (i) other PRRSV 1 or PRRSV 2 vaccine-like cases; (ii) reversion to virulence of attenuated vaccine strains; and (iii) low-virulence strains causing outbreaks in PRRSV-stable or PRRSV-free farms. Therefore, the viral evolution, origin and the difference between vaccine and field strains warrant further investigation.

In conclusion, this report is the first to describe an outbreak in Taiwan of a PRRSV 1 strain that shared a common evolutionary ancestor with a European PRRSV vaccine strain. This PRRSV 1 outbreak in Taiwan was attributable to the spread of vaccine viruses.

Supplementary Materials: The following are available online at http://www.mdpi.com/1999-4915/12/3/316/s1, Figure S1: Nucleotide sequence identity of the complete genome of PRRSV/NPUST-2789-3W-2/TW/2018 with that of other PRRSVs., Figure S2: Nucleotide sequence identity of ORF5 of PRRSV/NPUST-2789-3W-2/TW/2018 with that of other PRRSVs. 
Author Contributions: W.-H.L., K.K. and S.-Y.W.; methodology, W.-H.L.; software, C.-F.L. and C.-Y.Y.; validation, M.-T.C. and C.-N.L.; resources. W.-H.L. and K.K.; writing-original draft preparation, M.-T.C. and C.-N.L.; writing-review and editing. All authors have read and agreed to the published version of the manuscript.

Funding: This research received no external funding.

Acknowledgments: We thank the swine veterinary residents Bo-Rong Lian and Meng-Tien Wu and laboratory technician Ying-Xiu Lian for assistance with viral nucleic acid isolation, antibody detection and diagnosis.

Conflicts of Interest: The authors declare no conflict of interest.

\section{References}

1. Wensvoort, G.; Terpstra, C.; Pol, J.M.; ter Laak, E.A.; Bloemraad, M.; de Kluyver, E.P.; Kragten, C.; van Buiten, L.; den Besten, A.; Wagenaar, F.; et al. Mystery swine disease in The Netherlands: The isolation of Lelystad virus. Vet. Q. 1991, 13, 121-130. [CrossRef] [PubMed]

2. Collins, J.E.; Benfield, D.A.; Christianson, W.T.; Harris, L.; Hennings, J.C.; Shaw, D.P.; Goyal, S.M.; McCullough, S.; Morrison, R.B.; Joo, H.S.; et al. Isolation of swine infertility and respiratory syndrome virus (isolate ATCC VR-2332) in North America and experimental reproduction of the disease in gnotobiotic pigs. J. Vet. Diagn. Investig. 1992, 4, 117-126. [CrossRef] [PubMed]

3. Carman, S.; Sanford, S.E.; Dea, S. Assessment of seropositivity to porcine reproductive and respiratory syndrome (PRRS) virus in swine herds in Ontario-1978 to 1982. Can. Vet. J. 1995, 36, 776-777. [PubMed]

4. Adams, M.J.; Lefkowitz, E.J.; King, A.M.Q.; Harrach, B.; Harrison, R.L.; Knowles, N.J.; Kropinski, A.M.; Krupovic, M.; Kuhn, J.H.; Mushegian, A.R.; et al. Changes to taxonomy and the International Code of Virus Classification and Nomenclature ratified by the International Committee on Taxonomy of Viruses (2017). Arch. Virol. 2017, 162, 2505-2538. [CrossRef]

5. Murtaugh, M.P.; Elam, M.R.; Kakach, L.T. Comparison of the structural protein coding sequences of the VR-2332 and Lelystad virus strains of the PRRS virus. Arch. Virol. 1995, 140, 1451-1460. [CrossRef]

6. Nelsen, C.J.; Murtaugh, M.P.; Faaberg, K.S. Porcine reproductive and respiratory syndrome virus comparison: Divergent evolution on two continents. J. Virol. 1999, 73, 270-280. [CrossRef]

7. Wensvoort, G.; de Kluyver, E.P.; Luijtze, E.A.; den Besten, A.; Harris, L.; Collins, J.E.; Christianson, W.T.; Chladek, D. Antigenic comparison of Lelystad virus and swine infertility and respiratory syndrome (SIRS) virus. J. Vet. Diagn. Investig. 1992, 4, 134-138. [CrossRef]

8. Zimmerman, J.J.; Dee, S.A.; Holtkamp, D.J.; Murtaugh, M.P.; Stadejek, T.; Stevenson, G.W.; Torremorell, M.; Yang, H.; Zhang, J. Porcine Reproductive and Respiratory Syndrome Viruses (Porcine Arteriviruses). In Diseases of Swine, 11th ed.; Zimmerman, J.J., Karriker, L.A., Ramirez, A., Schwartz, K.J., Stevenson, G.W., Zhang, J., Eds.; Wiley-Blackwell: Hoboken, NJ, USA, 2019; pp. 685-708.

9. Murtaugh, M.P.; Genzow, M. Immunological solutions for treatment and prevention of porcine reproductive and respiratory syndrome (PRRS). Vaccine 2011, 29, 8192-8204. [CrossRef]

10. Nielsen, H.S.; Oleksiewicz, M.B.; Forsberg, R.; Stadejek, T.; Botner, A.; Storgaard, T. Reversion of a live porcine reproductive and respiratory syndrome virus vaccine investigated by parallel mutations. J. Gen. Virol. 2001, 82, 1263-1272. [CrossRef]

11. Marton, S.; Szalay, D.; Kecskemeti, S.; Forro, B.; Olasz, F.; Zadori, Z.; Szabo, I.; Molnar, T.; Banyai, K.; Balint, A. Coding-complete sequence of a vaccine-derived recombinant porcine reproductive and respiratory syndrome virus strain isolated in Hungary. Arch. Virol. 2019, 164, 2605-2608. [CrossRef]

12. Wang, A.; Chen, Q.; Wang, L.; Madson, D.; Harmon, K.; Gauger, P.; Zhang, J.; Li, G. Recombination between Vaccine and Field Strains of Porcine Reproductive and Respiratory Syndrome Virus. Emerg. Infect. Dis. 2019, 25, 2335-2337. [CrossRef] [PubMed]

13. Zhou, L.; Kang, R.; Yu, J.; Xie, B.; Chen, C.; Li, X.; Xie, J.; Ye, Y.; Xiao, L.; Zhang, J.; et al. Genetic Characterization and Pathogenicity of a Novel Recombined Porcine Reproductive and Respiratory Syndrome Virus 2 among Nadc30-Like, Jxa1-Like, and Mlv-Like Strains. Viruses 2018, 10, 551. [CrossRef] [PubMed]

14. Eclercy, J.; Renson, P.; Lebret, A.; Hirchaud, E.; Normand, V.; Andraud, M.; Paboeuf, F.; Blanchard, Y.; Rose, N.; Bourry, O. A Field Recombinant Strain Derived from Two Type 1 Porcine Reproductive and Respiratory Syndrome Virus (PRRSV-1) Modified Live Vaccines Shows Increased Viremia and Transmission in SPF Pigs. Viruses 2019, 11, 296. [CrossRef] [PubMed] 
15. Van Dooren, K. Denmark: New PRRS strain emerged. Pig Progress, 16 November 2019. Available online: https: //www.pigprogress.net/Piglets/Articles/2019/11/Denmark-New-PRRS-strain-emerged-in-July-499756E/.

16. Chen, N.; Cao, Z.; Yu, X.; Deng, X.; Zhao, T.; Wang, L.; Liu, Q.; Li, X.; Tian, K. Emergence of novel European genotype porcine reproductive and respiratory syndrome virus in mainland China. J. Gen. Virol. 2011, 92, 880-892. [CrossRef]

17. Iseki, H.; Takagi, M.; Kawashima, K.; Shibahara, T.; Kuroda, Y.; Tsunemitsu, H.; Yamakawa, M. Type 1 porcine reproductive and respiratory syndrome virus emerged in Japan. In Proceedings of the 22nd International Pig Veterinary Society Congress, Jeju, Korea, 10-13 June 2012; p. 978.

18. Kim, S.H.; Roh, I.S.; Choi, E.J.; Lee, C.; Lee, C.H.; Lee, K.H.; Lee, K.K.; Song, Y.K.; Lee, O.S.; Park, C.K. A molecular analysis of European porcine reproductive and respiratory syndrome virus isolated in South Korea. Vet. Microbiol. 2010, 143, 394-400. [CrossRef]

19. Nguyen, N.H.; Vuong, T.H.V.; Vo, T.H. PRRS in Vietnam and its diagnosis. J. Life Sci. 2015, 9, $272-276$.

20. Thanawongnuwech, R.; Amonsin, A.; Tatsanakit, A.; Damrongwatanapokin, S. Genetics and geographical variation of porcine reproductive and respiratory syndrome virus (PRRSV) in Thailand. Vet. Microbiol. 2004, 101, 9-21. [CrossRef]

21. Yoshii, M.; Kaku, Y.; Murakami, Y.; Shimizu, M.; Kato, K.; Ikeda, H. Polymerase chain reaction-based genetic typing of Japanese porcine reproductive and respiratory syndrome viruses. J. Vet. Diagn. Investig. 2004, 16, 342-347. [CrossRef]

22. Zhou, L.; Yang, H. Porcine reproductive and respiratory syndrome in China. Virus Res. 2010, 154, 31-37. [CrossRef]

23. Wu, H.-H.; Lin, W.-H.; Su, G.-S.; Lin, C.-N.; Chiou, M.-T. Comparison of viremia of type I and II porcine reproductive and respiratory syndrome virus in Taiwan. In 24th International Pig Veterinary Society Congress; Kirwan, P., Ed.; Pat Kirwan: Dublin, Ireland, 2016; p. 547.

24. Holtkamp, D.J.; Polson, D.D.; Torremorell, M.; Morrison, B.; Classen, D.M.; Becton, L.; Henry, S.; Rodibaugh, M.T.; Rowland, R.R.; Snelson, H.; et al. Terminology for classifying the porcine reproductive and respiratory syndrome virus (PRRSV) status of swine herds. Tierarztl Prax Ausg G Grosstiere Nutztiere 2011, 39, 101-112.

25. Lin, C.N.; Lin, W.H.; Hung, L.N.; Wang, S.Y.; Chiou, M.T. Comparison of viremia of type II porcine reproductive and respiratory syndrome virus in naturally infected pigs by zip nucleic acid probe-based real-time PCR. BMC Vet. Res. 2013, 9, 181. [CrossRef] [PubMed]

26. Lin, W.H.; Shih, H.C.; Wang, S.Y.; Lin, C.F.; Yang, C.Y.; Chiou, M.T.; Lin, C.N. Emergence of a virulent porcine reproductive and respiratory syndrome virus in Taiwan in 2018. Transbound. Emerg. Dis. 2019, 66, 1138-1141. [CrossRef] [PubMed]

27. Kumar, S.; Stecher, G.; Li, M.; Knyaz, C.; Tamura, K. MEGA X: Molecular Evolutionary Genetics Analysis across Computing Platforms. Mol. Biol. Evol. 2018, 35, 1547-1549. [CrossRef] [PubMed]

28. Lole, K.S.; Bollinger, R.C.; Paranjape, R.S.; Gadkari, D.; Kulkarni, S.S.; Novak, N.G.; Ingersoll, R.; Sheppard, H.W.; Ray, S.C. Full-length human immunodeficiency virus type 1 genomes from subtype C-infected seroconverters in India, with evidence of intersubtype recombination. J. Virol. 1999, 73, 152-160. [CrossRef] [PubMed]

29. Madsen, K.G.; Hansen, C.M.; Madsen, E.S.; Strandbygaard, B.; Botner, A.; Sorensen, K.J. Sequence analysis of porcine reproductive and respiratory syndrome virus of the American type collected from Danish swine herds. Arch. Virol. 1998, 143, 1683-1700. [CrossRef]

30. Chen, N.; Liu, Q.; Qiao, M.; Deng, X.; Chen, X.; Sun, M. Whole genome characterization of a novel porcine reproductive and respiratory syndrome virus 1 isolate: Genetic evidence for recombination between Amervac vaccine and circulating strains in mainland China. Infect. Genet. Evol. 2017, 54, 308-313. [CrossRef]

(C) 2020 by the authors. Licensee MDPI, Basel, Switzerland. This article is an open access article distributed under the terms and conditions of the Creative Commons Attribution (CC BY) license (http://creativecommons.org/licenses/by/4.0/). 